\title{
An investigation on dynamic characteristics of herringbone planetary gear system with torsional flexibility between the left and right teeth of the sun gear
}

\author{
Xiangyang $\mathrm{Xu}^{*}$, Hongwei Ge, Jijun Deng, Jibo Wang, and Renxiang Chen \\ School of Mechanotronics and Vehicle Engineering, Chongqing Jiaotong University, ChongQing 400074, China
}

Received: 24 April 2020 / Accepted: 30 August 2020

\begin{abstract}
Herringbone planetary gear system (HPGS) has high power density and complex structure. The torsional flexibility of the left and right teeth of the sun gear is closely related to the dynamic characteristics of the HPGS. In this research, considering the coordination conditions of both sides torsional stiffness and axial slide of the sun gear, a new dynamic model of the HPGS considering the meshing phase difference between left and right teeth of the sun gear is developed based on the lumped-parameter method, and the influence mechanism of torsional stiffness and axial sliding is studied. Moreover, the dynamic parameters and dynamic characteristics of the HPGS are analyzed in the case of varying torsoinal stiffness and axial slide. The results show that the torsional stiffness of left and right teeth and the axial slide of sun gear have significant impacts on the dynamic parameters and dynamic mesh force response. With the increase of the torsional flexibility (the decrease the torsional stiffness), the sun gear and planet gear meshing stiffness and the maximum tooth surface load are both increased on the left side (input side) and decreased on the right side, but the main peak values and peak frequencies of dynamic response on both sides of the $s$ - $p$ meshing pairs decrease significantly. In addition, when the sun gear slides toward the output side axially, meshing stiffness and dynamic mesh force response main peak values decreased on the left side (input side) and increased on the right side, but the main resonance peaks frequencies keep the same.
\end{abstract}

Keywords: Herringbone planetary gear / sun gear / torsional flexibility / meshing stiffness / dynamic characteristics

\section{Introduction}

With the increasing power of wind turbines, the aero-space equipment, large machine tools, etc., the herring-bone planetary gear system (HPGS) has become the main research fields of planetary gear transmission system for its distinctive advantages of more compact structure, higher power density and greater carrying capacity. The traditional dynamic characteristics design for general planetary gear transmission system is difficult to meet this type high power application requirements more and more. Thus, increasing the flexibility of this kind of system is an momentous research direction to obtain better load distribution and dynamic characteristics. Therefore, in the research of HPGS, it has great practical significance to study gears dynamic load when torsional flexibility of the two symmetrical sides of the herringbone sun gear is considered.

\footnotetext{
* e-mail: xxiangyang@hotmail.com
}

Currently, some scholars have conducted a series of researches on the dynamic characteristics of the HPGS. Lin, J., and Parker, R. G [1] proposed a planar dynamic model of planetary gears and summarized three vibration modes of plane vibration. Zhang and Zhu [2] developed a novel dynamic model of HPGS considering the meshing phase, and analyzed the influence of meshing phase on load sharing. Sondkar et al. [3] proposed a dynamic model of the HPGS, which includes the degrees of freedom of torsion, translation and rotation of rotating components, and found that the stagger on both sides has a significant effect on the dynamic response of HPGS. Bu et al. [4] studied the modal characteristics by establishing a generalized linear model of the HPGS with three plane degrees of freedom, simultaneously deriving a different relationship between planet deflections under the translation mode. Zhu [5] and $\mathrm{Xu}$ et al. [6] analyzed the dynamic characteristics and the load sharing performance by establishing a planetary gear dynamic model with flexible pins. Wang et al. [7] considered the effects of friction and multiple backlashes while taking axial vibration into consideration, then 
established a dynamic model of the herringbone gear set, and solved the dynamic equation with an example. Wang et al. [8] optimized the vibration of the herringbone gear and proposed a sixth-order polynomial transmission error function for reducing vibration and shock. Mo et al. [9,10] proposed a dynamic model of the HPGS, found that the torsional stiffness has a certain impact on the natural characteristics and load sharing. Liu [11] and Ren et al. [12] proposed a generalized model of the HPGS, which can be used to analyze the variable process and the effect of manufacturing errors on the dynamic floating performance of the system. Chen and Shao [13] considering gear tooth errors, and developed a dynamic model of planet gear, which is used to analyze the meshing stiffness of mesh pairs, and employed in any situation to analyze the effects of load distribution and load static transmission errors. Wang and Lim et al. [14] built a numerical model and discussed the effect of gear parameters set for the dynamic response. Miyoshi [15] and Sanchez [16] developed an analytical calculation method, which is used to calculate the results of the load distribution and the contact stress with a lateral contact ratio greater than two. Cooley and Parker [17] predicted the development and technical difficulties of planetary gear dynamics and vibration. But, due to the complexity symmetrical structure of HPGS, most scholars in the above studies believe that the dynamic characteristics of the two symmetrical sides of the herringbone gear are the same. Actually, the carrier and sun gear would twist during the operation of HPGS. And the torsion of the sun gear under load will cause the flexible dislocation between the two symmetrical sides of the sun gear. This torsional flexibility of sun gear will have a direct impact on the dynamic characteristics of the HPGS. In addition, due to bearing installation clearance, manufacturing and installation errors, the meshing center points of the single helical gears on both sides of the sun gear and the planetary gear are not in the same vertical plane of geometric center. These effects cause the axial sliding between left and right meshing centers, which will change meshing stiffness and dynamic mesh force response.

In this investigation, a dynamic model of the HPGS including torsional flexibility between two symmetrical sides teeth of the sun gear is developed, based on the lumped-parameter method considering the torsional flexibility and the axial slide of the both sides teeth of the sun gear. Then, the influences of change of torsional flexibility and axial slide of the both sides teeth of the sun gear on the dynamic parameters and dynamic characteristics for the HPGS are discussed.

\section{Dynamic model of the HPGS}

\subsection{Basic parameters and dynamic model of the HPGS}

A typical HPGS is shown in Figure 1. Blades are used as input to provide power for the transmission system. And the generator sets convert kinetic energy into electrical energy. The input torque is transferred to the planetary gears from the carrier, afterwards to sun gear shaft by

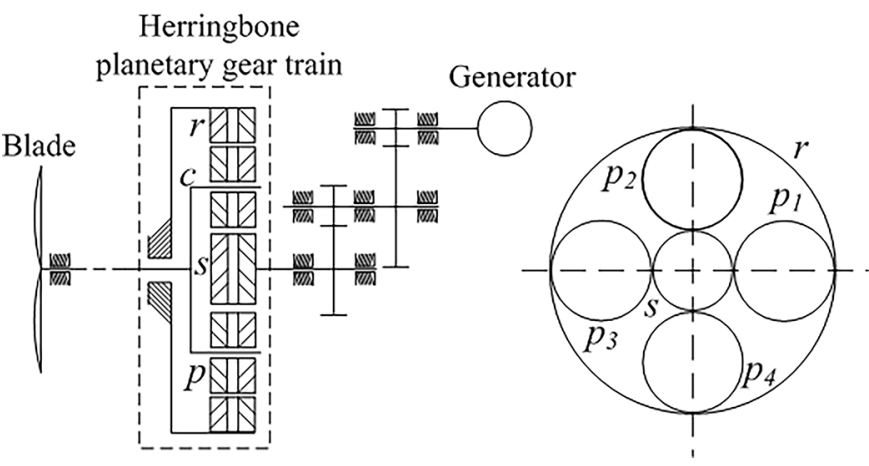

Fig. 1. Schematic diagram of HPGS.

Table 1. Structural parameters of HPGS.

\begin{tabular}{llll}
\hline Parameters & $p_{n}$ & \multicolumn{1}{c}{$s$} & $r$ \\
\hline Teeth number & 37 & 21 & 95 \\
Normal module/mm & 15 & 15 & 15 \\
Normal pressure angle/Degrees & 25 & 25 & 25 \\
Face width/mm & 175 & 185 & 185 \\
Helix angle/Degrees & 8 & 8 & 8 \\
Relief groove width of sun gear/mm & 50 & 40 & 40 \\
\hline
\end{tabular}

herringbone planetary train, then to parallel transmission stage by the sun gear shaft, and finally to the generator set. The rated input power of the HPGS are 1.5 MW. The material of all gears is $20 \mathrm{CrMnMo6}$. And the surfaces of all gears are carburized. The symbol of the HPGS is defined as follows: $r$ denotes the ring gear, and $c$ means the carrier, and $s$ represents the sun gear, and $p_{n}$ represents the $n$th planet gears, of which $(n=1,2,3,4)$. When the subscript $i$ equals $L$, it means the left side of the herringbone gear. When the subscript $i$ equals $R$, it indicates the right side of the herringbone gear. The structural parameters of the research object are illustrated in Table 1.

Dealing with the intermediate shaft section of herringbone gear is a very critical issue. In the process of dynamic modeling of the herringbone gear transmission system at home and abroad, in order to more accurately simulate the coupling between the structures, the herringbone gear is equivalent to the double helical gears on the left and right sides based on the three-piece model $[18,19]$. Under the beam theory that only considers torsion, a herringbone gear is divided into three pieces: same inner diameter on left and right sides gear segment, and a connecting structure, which spans from gear face mid-point from the left side $O_{L}$ to the right side $O_{R}$. The mass and moment of inertia concentrate on the adjacent nodes $O_{L}$ and $O_{R}$ respectively. Relief groove part is equivalent to the torsion beam elements with two nodes. The joints nodes $O_{L}$ and $O_{R}$ are at the beginning and end of the beam element respectively. The equivalent support stiffness and damping of the left and right support bearings of herringbone gear act on two nodes $O_{L}$ and $O_{R}$ respectively. Here, the relevant dynamic characteristic 


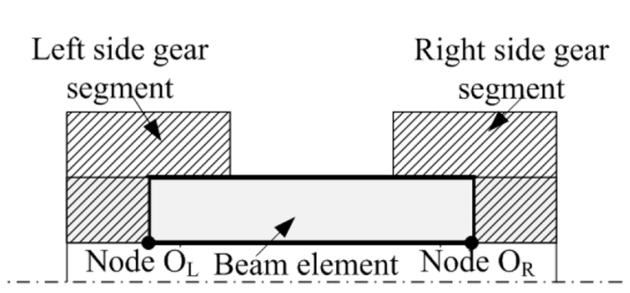

(a)

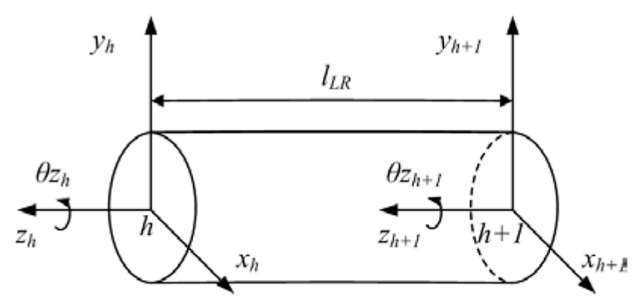

(b)

Fig. 2. Equivalent model of herringbone gear middle segment. (a) Three-piece model of herringbone gear. (b) Torsion beam element.

parameters are obtained by changing the torsional flexibility of the both sides tooth of the sun gear. $l_{L R}$ is the distance from point $O_{L}$ to $O_{R}$, which is the distance between the centers of the left and right single helical gears, and $l_{L T}$ is the distance from point $O_{L}$ to $O_{T}$, which is $657.5 \mathrm{~mm}$, and $l_{R T}$ is the distance from point $O_{R}$ to $O_{T}$, which is $432.5 \mathrm{~mm}$. Figure 2 illustrates equivalent model of herringbone gear middle segment. Figure 2a shows three-piece model of herringbone gear. Figure $2 \mathrm{~b}$ illustrates the schematic diagram of the beam element.

According to Figure 2, relative torsion and bending angle can be obtained by torsion beam element. Sun gear and planet gears are regarded as two single helical gears connected together, and each helical gear owns four degrees of freedom, comprising translational movements in the three directions of $x, y$, and $z$ axes, and a rotation around the $z$-axis. Therefore, only relative torsion angle is considered in the dynamic model. And relative torsion angle is inversely proportional to the torsional stiffness of the equivalent middle shaft segment $l_{L R}$, the parameter $\phi_{L j}$ is the relative torsion angle of the left single helical gear. Correspondingly, $\phi_{R j}$ is the relative torsion angle of the right side. The relative torsion angle $\phi_{L R j}$ of two single helical gears between $\phi_{L j}$ and $\phi_{R j}$ of the sun gear is expressed as equation (1).

$$
\phi_{L R j}=\int_{l_{L R}} \frac{T_{s}}{K_{j}} d l_{h}
$$

where $T_{s}$ denotes the external load on the sun gear, torsional stiffness $K_{j}=G_{j} I_{j}$, and the shear elastic modulus $G=E /(2(1+v)), E$ is the elastic modulus, $E=206 \mathrm{Gpa}$, and $v=0.3, I_{j}$ is the torsional moment of inertia.

According to equation (1), when the sun gear is applied with a constant external load, the torsional flexibility of the two symmetrical sides of the sun gear is determined by $K_{j}$ and $l$. Of course, the axial slide of the sun gear will affect the torsional flexibility between the both sides teeth of the sun gear.

The displacement of the beam element in the local coordinate system is illustrated in Figure 2. The focus of this article is to consider the influence of torsional flexibility, and the torsional flexibility is mainly investigated here. Therefore, only four degrees of freedom are considered in the single helical gear, comprising translational movements in the three directions of $x, y$, and $z$ axes, and a rotation around the $z$-axis. And the specific expression of the displacement vector of the equivalent double-helical gear system is shown in equation (2).

$$
q_{h}=\left(x_{h}, y_{h}, z_{h}, \theta_{z h}, x_{h+1}, y_{h+1}, z_{h+1}, \theta_{z h+1}\right)^{T} .
$$

The mass and moment of inertia of each part in the equivalent double-helical gear system are distributed according to the principle that the total mass, the total moment of inertia and the position of the mass center are not changed, and are aggregated to the adjacent nodes $L$ and $R$, respectively. The equivalent support stiffness of the left and right support bearings of herringbone gear are respectively, and acts on node $L$ and node $R$. Both the beam element mass matrix $M_{i e}(i=s, p)$ and the stiffness matrix $K_{i e}(i=s, p)$ are presented as block sub-matrices [18].

$$
\begin{gathered}
M_{i e}=\left[\begin{array}{cc}
M_{i e}{ }^{11} & 0 \\
0 & M_{i e}{ }^{22}
\end{array}\right] \\
K_{i e}=\left[\begin{array}{cc}
K_{i e}{ }^{11} & 0 \\
0 & K_{i e}{ }^{22}
\end{array}\right] .
\end{gathered}
$$

The specific form of each sub-matrix of the mass matrix $M_{i e}$ is as follows:

$$
\begin{aligned}
M_{i e}{ }^{11}= & {\left[\begin{array}{cccc}
m_{L s} & 0 & 0 & 0 \\
0 & m_{L s} & 0 & 0 \\
0 & 0 & m_{L s} & 0 \\
0 & 0 & 0 & \frac{I_{L s}}{r_{b s}^{2}}
\end{array}\right] } \\
M_{i e}{ }^{22}= & {\left[\begin{array}{cccc}
m_{R s} & 0 & 0 & 0 \\
0 & m_{R s} & 0 & 0 \\
0 & 0 & m_{R s} & 0 \\
0 & 0 & 0 & \frac{I_{R s}}{r_{b s}^{2}}
\end{array}\right] }
\end{aligned}
$$

where $m_{L s}$ denotes the mass of the left single helical gears of the sun gear, and $m_{R s}$ represents the mass of the right single helical gear of the sun gear, $r_{b s}$ is the base circle radius of sun gear, $I_{L s}$ is moment of inertia of the left single helical gears of the sun gear, $I_{R s}$ is moment of inertia of the right single helical gears of the sun gear.

Using the same method, the specific form of the stiffness matrix $K_{i e}$ can be obtained. In order to save space, it will not be listed here. 


\subsection{Meshing stiffness of HPGS considering the meshing phase}

According to the principle of elastic potential energy with considering the torsional flexibility of two sides teeth of sun gear, and comprehensive consideration of the effects of various factors on deformation, the single tooth meshing stiffness of $s-p$ meshing pairs and $r-p$ meshing pairs can be expressed as:

$$
\begin{aligned}
& K_{m}= \\
& \frac{1}{\left(1 / K_{b 1}+1 / K_{s 1}+1 / K_{a 1}+1 / K_{b 2}+1 / K_{s 2}+1 / K_{a 2}+1 / K_{h z}\right)}
\end{aligned}
$$

where $K_{b}, K_{s}, K_{a}$ are the stiffness of deformation of bending, shear and axial, respectively, and $K_{h z}$ is the stiffness of Hertz contact, and subscript 1 and 2 represent driving and driven gear, respectively.

There are three kinds of meshing phase differences in general planetary gears: $s-p$ mesh, $r-p$ mesh and between $s-p$ and $r-p$ mesh [6]. But, the herringbone planetary gear transmission has four meshing phases when torsional flexibility is considered. They are defined as the phase coefficient $\lambda_{s n}, \lambda_{r n}, \lambda_{r s}^{n}$ and $\lambda_{L R j}$ represent the relative phase of the $n$th to the first $s-p$ meshing pair, the $n$th to the first $r-p$ meshing pairs, the $n$th $s-p$ meshing pairs to the $n$th $r-p$ meshing pair and left $s-p$ meshing pair to right $s-p$ meshing pair, respectively. $\lambda_{L R j}$ is between left side and right side phasing difference caused by relative torsion angle $\phi_{L R j}$.

$\Delta t$ is the time interval of the $n$th to the first $s$ - $p$ meshing pair, $T_{m}$ denotes the mesh period of all phases.

$$
\begin{gathered}
\Delta t_{s n}=\lambda_{s n} T_{m} \\
T_{m}=\frac{2 \pi}{\left(\omega_{s}-\omega_{c}\right) z_{s}} .
\end{gathered}
$$

In this dynamic model, $\phi_{n}$ represents the angle of the $n$th to the first planet gear on the right side. As the right sun gear rotates angle $\phi_{n}$ relative to the carrier, the right side $n$th planet gear moves to the position right belonging to the right side first planet gear, and the expression of the required time $t$ is shown by the following:

$$
t=\frac{\phi_{n}}{\omega_{s}-\omega_{c}}
$$

Uniting the equation (6) and the equation (7):

$$
\left(\lambda_{s n}+p_{n}\right) T_{m}=\frac{\phi_{n}}{\omega_{s}-\omega_{c}}
$$

where $p_{n}$ is an integer, and the expression of $\lambda_{s n}$ can be obtained by combining the equations (6) and (7).

$$
\lambda_{s n}= \pm \operatorname{dec}\left(\frac{z_{s} \phi_{n}}{2 \pi}\right)
$$

where $\operatorname{dec}(A)$ represents the decimal part of $A$, so $\lambda_{s n}=\lambda_{r n}$ and $\lambda_{r s}^{(n)}=\lambda_{r s}^{(1)}$ can be derived.

$$
\begin{gathered}
\lambda_{r n}= \pm \operatorname{dec}\left(\frac{z_{r} \phi_{n}}{2 \pi}\right) \\
\lambda_{r s}=\frac{N_{r} T}{P_{b}}
\end{gathered}
$$

where $N_{r} T$ is the distance between $r$-pn initial meshing position point $\mathrm{T}$ and meshing pitch point $N_{r}$ when the starting point of $s-p_{n}$ meshing is at meshing pitch point $N_{\mathrm{s}}$, $P_{b}$ is the planetary gear pitch.

When considering torsional flexibility of the both sides teeth of the sun gear, $\left(\phi_{n}+\phi_{L R j}\right)$ means the actual rotation angle of the left side sun gear relative to the carrier, and the phase coefficient $\lambda_{L R j}$ is shown by the following:

$$
\lambda_{L R j}= \pm \operatorname{dec}\left(\frac{z_{s} \phi_{L R j}}{2 \pi}\right) .
$$

According to the equation (11), at any time $t$, the instantaneous total contact line length of single helical gear pair in herringbone gear is $L(t)$ as shown by the following:

$$
L(t)=\sum_{n=1}^{n} L_{n}(t) .
$$

Then, according to the equation (12), the expression of tooth meshing stiffness $k(t)$ can be derived by the following:

$$
k(t)=k_{0} L(t)
$$

where $k_{0}$ is the meshing stiffness per unit contact line length.

Therefore, considering the torsional flexibility of the both sides tooth of the sun gear, the expression of meshing stiffness of the $s-p$ meshing pairs is shown as:

$$
\begin{gathered}
k_{L s 1}(t)=k_{0 L s} L\left(t_{L}\right) \\
k_{L s n}(t)=k_{0 L s} L\left(t-\lambda_{s n} T_{m}\right) \\
k_{R s 1}(t)=k_{0 R s} L(t) \\
k_{R s n}(t)=k_{0 R s} L\left(t-\left(\lambda_{s n}+\lambda_{L R j}\right) T_{m}\right) .
\end{gathered}
$$

This article only considers the torsional flexibility of the both sides tooth of the sun gear, so there is no influence on $r-p$ meshing pairs, and the expression of its meshing stiffness is shown as:

$$
\begin{array}{r}
k_{L r 1}(t)=k_{R r 1}(t)=k_{0 r n} L\left(t-\lambda_{r s} T_{m}\right) \\
k_{L r n}(t)=k_{R r n}(t)=k_{0 r n} L\left(t-\left(\lambda_{r n}+\lambda_{r s}\right) T_{m}\right)
\end{array}
$$

where $\lambda_{r s}$ is meshing phase difference between $s-p$ mesh and $r-p$ mesh. 


\subsection{Dynamic equations of the HPGS}

The dynamic model of HPGS is shown in Figure 3, Three coordinate systems are established in the above model. $O X Y Z$ expresses a static coordinate system. And their origin is the rotation center of the carrier. The $x$-axis through the first planet's rotation center and uses the rotation center of the carrier as the origin, which rotates at a constant speed around the $z$-axis with the angular velocity $\omega_{c}$ of the carrier. $o_{n} x_{n} y_{n} z_{n}$ is a moving coordinate system. The difference between $o_{n} x_{n} y_{n} z_{n}$ is that $o_{n} x_{n} y_{n} z_{n}$ rotates at a constant speed with carrier around the $z_{n}$-axis, using the rotation center of each planetary gear as the origin.

In this dynamic model, herringbone sun gear and planet gears are regarded as two single helical gears connected together. Left and right sides of herringbone gears are assumed identical in geometry expect the hand of the teeth is opposite with respect to each other. In an actual herringbone planetary gear system, left and right sides of gears and carrier are either one-piece (for planet and sun gears) or connected rigidly (for the ring gear and carrier).

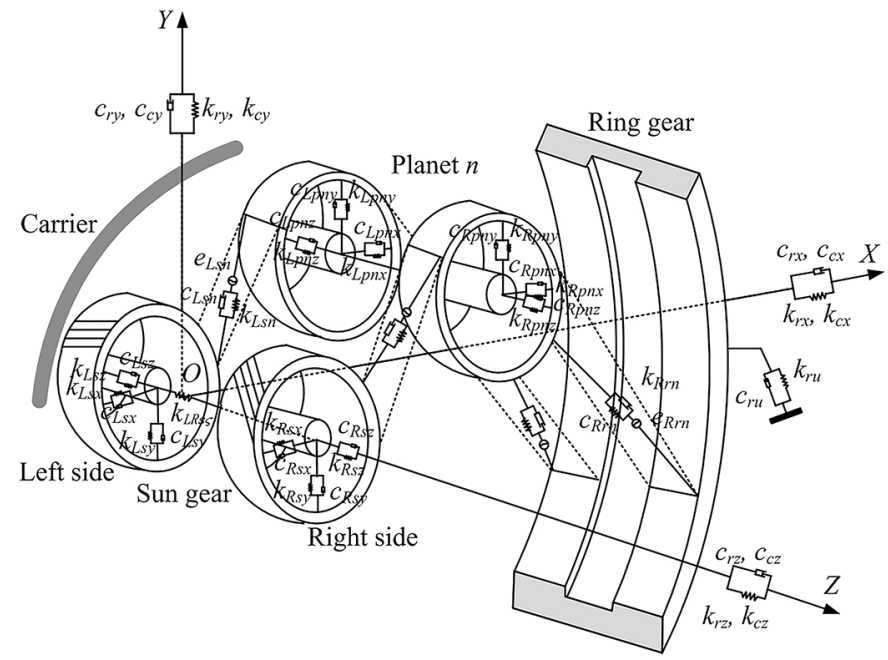

Fig. 3. Dynamic model of the HPGS.
Sun gear, planet gears and ring gear are regarded as two single helical gears connected together, and each helical gear owns four degrees of freedom, comprising translational movements in the three directions of $x, y$, and $z$ axes, and a rotation around the $z$-axis. In addition, the following assumptions are made in the dynamic model: (a) without considering the energy loss, the input torque can be directly applied on the carrier, and the load torsion can directly applied on the sun gear. (b) It is assumed that the left and right sides of herringbone gear are geometrically symmetrical, and the rotation of teeth is opposite to each other. They are staggered by a certain angle with torsional flexibility of relief groove. (c) It is difficult to obtain the damping coefficient, damping of the system is represented by a constant modal damping, and the friction effect of gear pair meshing and the friction between other parts are ignored. According to the equation (16), the displacement vector $q$ of the system is obtained. Moreover, the model of the transmission system contains $8(N+3)$ degrees of freedom in total.

$q=\left(x_{i s}, y_{i s}, z_{i s}, \theta_{i \mathrm{~s}}, x_{i n}, y_{i n}, z_{i n}, \theta_{i n}, x_{i r}, y_{i r}, z_{i r}, \theta_{i r}, x_{i c}, y_{i c}, z_{i c}, \theta_{i c}\right)^{T}$

where $x, y$, and $z$ denote the lateral, longitudinal and axial displacements of the component, respectively. The parameter $\theta$ denotes the angular displacement, the subscript is denotes the sun gear, the subscript in denotes the $n$th planet gear, $r$ is the ring gear, and $c$ is the carrier.

Figure 4 illustrates the relative positional relationship of the components of the HPGS. The left side relative displacements of the $s-p$ and $r$ - $p$ meshing pairs perpendicular to the tooth surface can be obtained from Figure 4 . The right side relative displacements are symmetrical with left side.

Before calculating the relative displacement of the herringbone tooth, this paper takes the herringbone gear quivalent to two identical single helical gears on the left and right sides respectively. Therefore, the relative displacement of the left and right sides needs to be calculated separately. Taking the calculation of the left-side relative displacement of the sun gear and the planet gear as an example, the solution process will be introduced in detail.

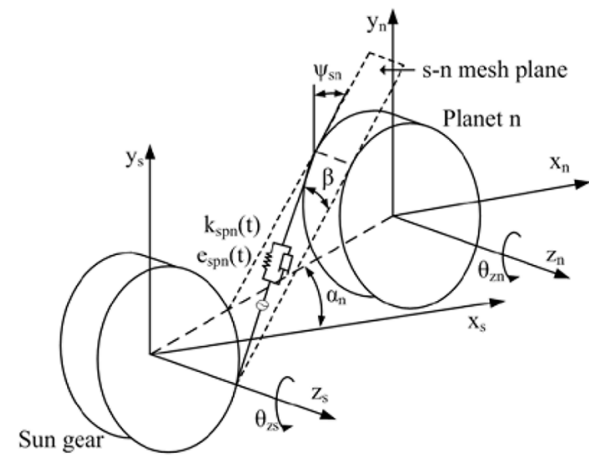

(a)

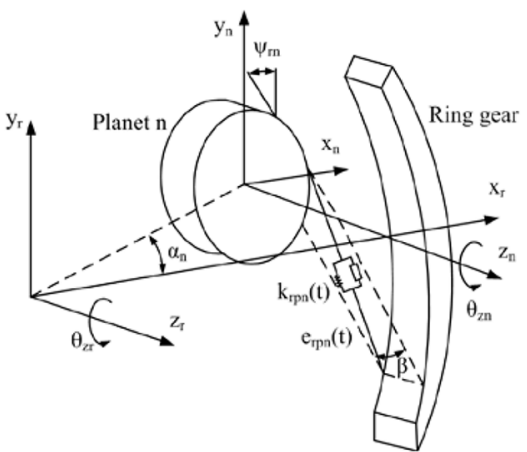

(b)

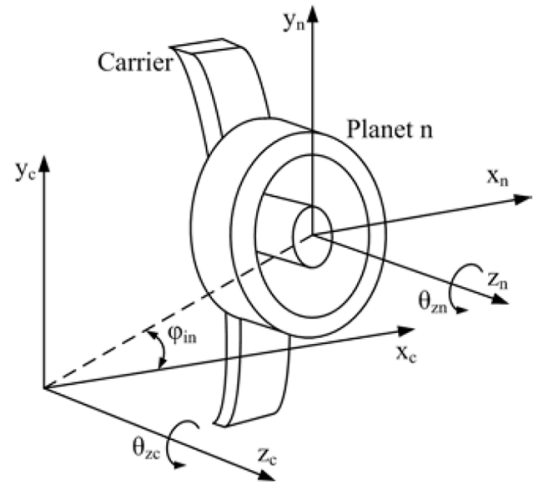

(c)

Fig. 4. Left side positional relationship diagram of the HPGS. (a) Sun-planet mesh. (b) Ring-planet mesh. (c) Carrier-planet. 
Projecting the displacement of the sun gear and planetary gear in the $x$ direction onto the meshing plane $s$ - $n$ to obtain $x_{L s} \sin \psi_{s n}$ and $x_{L n} \sin \psi_{s n}$. Since the sun gear and the planet gear rotate in opposite directions, the displacement projections of the sun gear and the planet gear on the meshing line in the $x$ direction are $\left(-x_{L s} \sin \psi_{s n} \cos \beta_{L b}\right)$ and $x_{L n} \sin \psi_{s n} \cos \beta_{L b}$, respectively. Therefore, the comprehensive relative displacement of the sun gear and planetary gear in the $x$ direction is $\left(x_{L n}-x_{L s}\right)$ $\sin \psi_{s n} \cos \beta_{L b}$. Using the same method, the projections $\left(y_{L s}-y_{L n}\right) \cos \psi_{s n} \cos \beta_{L b}$ and $\left(z_{L s}+z_{a h}-z_{L n}\right) \sin \beta_{L b}$ of the displacements in the $y$-direction and $z$-direction of the sun gear and planetary gears on the meshing line can be obtained, respectively. Due to torsion flexibility and manufacturing and installation errors, the projection $\left[r_{b s}\left(\theta_{L s}+\phi_{h}\right)+r_{b p} \theta_{L n}\right] \cos \beta_{L b}$ of the torsional freedom and the transmission error $e_{L s n}$ need to be added. Therefore, we can easily get the equation (17a).

Using the same method, we can obtain the following equations (18) and (19). Therefore, $\delta_{L s n}$ and $\delta_{L s n}$ represent the relative meshing displacement of the $s-p$ mesh in the direction normal to tooth surface such that [18]:

$$
\begin{aligned}
\delta_{L s n}=[ & \left(y_{L s}-y_{L n}\right) \cos \psi_{s n}+\left(x_{L n}-x_{L s}\right) \sin \psi_{s n} \\
& \left.+r_{b s}\left(\theta_{L s}+\phi_{h}\right)+r_{b p} \theta_{L n}\right] \cos \beta_{L b} \\
& +\left(z_{L s}+z_{a h}-z_{L n}\right) \sin \beta_{L b}-e_{L s n} \\
\delta_{R s n}=[ & \left(y_{R s}-y_{R n}\right) \cos \psi_{s n}+\left(x_{R n}-x_{R s}\right) \sin \psi_{s n} \\
& \left.+r_{b s} \theta_{R s}+r_{b p} \theta_{R n}\right] \cos \beta_{R b} \\
& +\left(z_{L n}+z_{a h}-z_{L s}\right) \sin \beta_{R b}-e_{R s n}
\end{aligned}
$$

Relative displacement between ring gear and planet gears is:

$$
\begin{aligned}
\delta_{i n r}= & {\left[\left(y_{i n}-y_{i r}\right) \cos \psi_{s n}+\left(x_{i r}-x_{i n}\right) \sin \psi_{r n}-r_{b p} \theta_{i n}+r_{b r} \theta_{i r}\right] } \\
& \cos \beta_{i b}+\left(z_{i n}-z_{i r}\right) \sin \beta_{i b}-e_{i n r} .
\end{aligned}
$$
is:

Relative displacement of the planet gear to the carrier

$$
\begin{gathered}
\delta_{i c n x}=x_{i c}-x_{i n}-r_{i c} \theta_{i c} \sin \varphi_{i n} \\
\delta_{i c n y}=y_{i c}-y_{i n}+r_{i c} \theta_{i c} \cos \varphi_{i n} \\
\delta_{i c n z}=z_{i c}-z_{i n} \\
\delta_{i c n c}=\left(x_{i n}-x_{i c}\right) \sin \phi_{i n}+\left(y_{i c}-y_{i n}\right) \cos \phi_{i n}+r_{i c} \theta_{i c}
\end{gathered}
$$

where $\psi_{s n}=\varphi_{s p}-\alpha_{n}, \quad \psi_{r n}=\varphi_{r p}+\alpha_{n}, \quad r_{b s}, \quad r_{b p}$ and $r_{b r}$ represent the radius of the base circle of the sun, planet, and ring gears, respectively, and $r_{c}$ denotes the distance from the center of planet gear to rotation center of carrier, and $\varphi$ represents the transverse pressure angle, $\alpha_{n}$ represents the position angle belonging to $n$th planet gears, $\beta_{i b}$ denotes the helix angle of the single helical gear on two symmetrical sides of the herringbone gears, and $z_{a}$ represents the axial slide of the sun gear, $e_{i s n}$ and $e_{i n r}$ denote respectively the transmission error on the $n$th $s-p$ and $r-p$ meshing pairs.

To reflect the dynamic characteristics of the HPGS more truly, this model considers the torsional flexibility of the both sides tooth of the sun gear. The dynamic equations of the single helical gear on the left and right sides of the sun gear can be determined as follows:

$$
\begin{aligned}
& m_{L s}\left(\ddot{x}_{L s}-2 \omega_{c} \dot{y}_{L s}-\omega_{c}^{2} x_{L s}\right)+k_{L R s x}\left(x_{L s}-x_{R s}\right) \\
& +\left(k_{L s x} x_{L s}+c_{L s x}\left(\dot{x}_{i s}-\omega_{c} y_{L s}\right)\right) \\
& -\sum_{n=1}^{N}\left[\left(k_{L s n} \delta_{L s n}+c_{L s n} \dot{\delta}_{L s n}\right) \sin \left(\varphi_{L n}-\alpha_{L s p}\right)\right] \\
& \quad \cos \beta_{L b}=0
\end{aligned}
$$

$$
\begin{aligned}
& m_{L s}\left(\ddot{y}_{L s}+2 \omega_{c} \dot{x}_{L s}-\omega_{c}^{2} y_{L s}\right)+k_{L R s y}\left(y_{L s}-y_{R s}\right) \\
& +\left(k_{L s y} y_{L s}+c_{L s y}\left(\dot{y}_{L s}+\omega_{c} x_{L s}\right)\right. \\
& +\sum_{n=1}^{N}\left[\left(k_{L s n} \delta_{L s n}+c_{L s n} \dot{\delta}_{L s n}\right) \cos \left(\varphi_{L n}-\alpha_{L s p}\right)\right] \\
& \quad \cos \beta_{L b}=0 \\
& m_{L s} \ddot{z}_{l s}+k_{L R s z}\left(z_{L s}-z_{R s}\right)+\left(k_{L s z} z_{L s}+c_{L s z} \dot{z}_{L s}\right) \\
& +\sum_{n=1}^{N}\left[\left(k_{L s n} \delta_{L s n}+c_{L s n} \dot{\delta}_{L s n}\right)\right] \sin \beta_{L b}=0
\end{aligned}
$$

$$
\begin{aligned}
& \frac{I_{L s}}{r_{b s}^{2}} \ddot{u}_{L s}+k_{L R s u}\left(u_{L s}-u_{R s}\right)+\left(k_{L s u} u_{L s}+c_{L s u} \dot{u}_{L s}\right) \\
& +\sum_{n=1}^{N}\left(k_{L s n} \delta_{L s n}+c_{L s n} \dot{\delta}_{L s n}\right) \\
& \quad \cos \beta_{L b}=\frac{T_{D}}{2 \cdot r_{b s}}
\end{aligned}
$$

$$
\begin{gathered}
m_{R s}\left(\ddot{x}_{R s}-2 \omega_{c} \dot{y}_{R s}-\omega_{c}^{2} x_{R s}\right)-k_{L R s x}\left(x_{R L s}-x_{R s}\right) \\
+\left(k_{R s x} x_{R s}+c_{R s x}\left(\dot{x}_{R s}-\omega_{c} y_{R s}\right)\right) \\
-\sum_{n=1}^{N}\left[\left(k_{R s n} \delta_{R s n}+c_{R s n} \dot{\delta}_{R s n}\right) \sin \left(\varphi_{R n}-\alpha_{R s p}\right)\right] \\
\quad \cos \beta_{R b}=0 \\
m_{R s}\left(\ddot{y}_{R s}+2 \omega_{c} \dot{x}_{R s}-\omega_{c}^{2} y_{R s}\right)-k_{L R s y}\left(y_{L s}-y_{R s}\right) \\
\quad+\left(k_{R s y} y_{R s}+c_{R s x}\left(\dot{y}_{R s}+\omega_{c} x_{R s}\right)\right) \\
\quad+\sum_{n=1}^{N}\left[\left(k_{R s n} \delta_{R s n}+c_{R s n} \dot{\delta}_{R s n}\right)\right. \\
\left.\quad \cos \left(\varphi_{R n}-\alpha_{R s p}\right)\right] \cos \beta_{R b}=0 \\
m_{R s} \ddot{z}_{R s}-k_{L R s z}\left(z_{L s}-z_{R s}\right)+\left(k_{R s z} z_{L R s}+c_{R s z} \dot{z}_{R s}\right) \\
+\sum_{n=1}^{N}\left[\left(k_{R s n} \delta_{R s n}+c_{R s n} \dot{\delta}_{R s n}\right)\right] \sin \beta_{R b}=0
\end{gathered}
$$




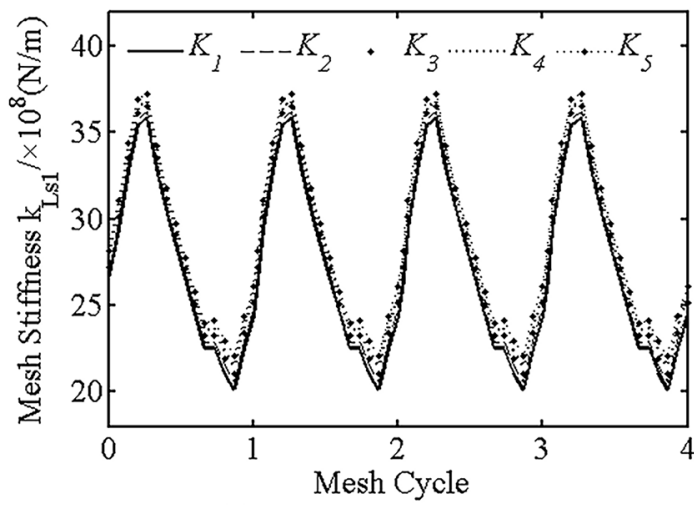

(a)

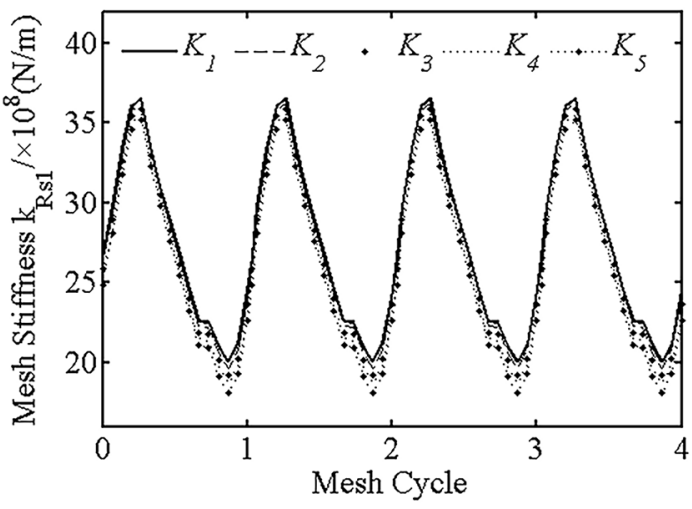

(b)

Fig. 5. Left and right sides meshing stiffness of the $s$ - $p$ mesh with changing torsional stiffness. (a) Left side $s-p$ mesh cycle. (b) Right side $s$ - $p$ mesh cycle.

$$
\begin{aligned}
& \frac{I_{R s}}{r_{b s}^{2}} \ddot{u}_{L s}-k_{L R s u}\left(u_{L s}-u_{R s}\right)+\left(k_{R s u} u_{R s}+c_{R s u} \dot{u}_{R s}\right) \\
& \quad+\sum_{n=1}^{N}\left(k_{R s n} \delta_{R s n}+c_{R s n} \dot{\delta}_{R s n}\right) \cos \beta_{R b}=\frac{T_{D}}{2 \cdot r_{b s}}
\end{aligned}
$$

where $m_{L s}$ denotes the mass of the left single helical gears of the sun gear, and $m_{R s}$ represents the mass of the right single helical gear of the sun gear, $\varsigma$ is the three translational motions of freedom along the $x, y$ and $z$ directions, and a rotation around $z$-axis, $T_{D}$ represents input and output torque, $k_{L R s \zeta}$ is the stiffness on the $\varsigma$ direction between the two symmetrical sides single helical gear of the sun gear, $k_{i s \varsigma}$ and $c_{i s \varsigma}$ denote the support stiffness and support damping of the sun gear along the $\varsigma$ direction, $k i s n$ and $c_{i s n}$ denote the meshing stiffness and mesh damping between the $n$th planet gears and the sun gear, respectively.

Equations of motion of the components ring gear, carrier and the planets can be derived in a similar way. The $8(N+3)$ DOF coupled dynamic equations can then be derived through the coordination of the rotation angles and the balance of the torques. The dynamic equations of the HPGS are arranged into a matrix modus as follows:

$$
\begin{aligned}
\mathbf{M} \ddot{q}+ & \left(\mathbf{C}_{b}+\mathbf{C}_{m}+\omega_{c} \mathbf{G}\right) \dot{q}+\left(\mathbf{K}_{b}+\mathbf{K}_{m}(t)-\omega_{c}^{2} \mathbf{K}_{\omega}\right) q \\
& =\mathbf{T}(t)+F(t)
\end{aligned}
$$

where $\mathbf{C}_{b}$ is the matrix of support damping, and $\mathbf{C}_{m}$ represents the matrix of meshing damping, and $\mathbf{G}$ denotes the matrix of gyroscopic, and $\mathbf{M}$ represents the mass matrix belonging to the system, and $\omega_{c}$ expresses the angular speed belonging to the carrier, and $\mathbf{K}_{b}$ denotes matrix of the support stiffness, and $\mathbf{K}_{m}$ means the matrix of meshing stiffness, and $\mathbf{K}_{\omega}$ represents the matrix of the centripetal stiffness, and $\mathbf{T}$ expresses the torque vector, and $F(t)$ is individual force vector caused by transmission error and time-varying meshing stiffness.

The equations of the dynamic mesh force response of sun gear and planet gear, ring gear and planet gear as follows:

$$
F_{i s p}=k_{i s n} \delta_{i s n}+c_{i s n} \dot{\delta}_{i s n}
$$

$$
F_{i r p}=k_{i r n} \delta_{i r n}+c_{i r n} \dot{\delta}_{i r n}
$$

where $F_{i s p}$ denotes the dynamic mesh force response of $s-p$ meshing pairs, and $F_{\text {irp }}$ represents the dynamic mesh force response of $r$ - $p$ meshing pairs, and $k_{i s n}$ represents the meshing stiffness of $s$ - $p$ meshing pairs, and $k_{i r n}$ denotes the meshing stiffness $r-p$ meshing pairs, and $c_{i s n}$ is the mesh damping of $s-p$ meshing pairs, and $c_{i r n}$ is the mesh damping of $r-p$ meshing pairs.

\section{Influence analysis of meshing parameters}

\subsection{Influence on the time-varying meshing stiffness}

In order to study the influence of torsional stiffness of sun gear on meshing stiffness, a series of meshing stiffness $k_{i s n}$ of the system can be obtained under different torsional stiffness according to equations (14) and (15). The initial torsional stiffness between the both sides teeth of the sun gear is $5.2 \times 10^{7} \mathrm{~N} \mathrm{~m}^{2}$ based on the equation (1). Here, the variation of torsional stiffness changes from $5.2 \times 10^{7} \mathrm{Nm}^{2}$ to $4.0 \times 10^{7} \mathrm{Nm}^{2}$. The torsional stiffness $K_{1}, K_{2}, K_{3}, K_{4}, K_{5}$ are $5.2 \times 10^{7} \mathrm{Nm}^{2}, 4.9 \times 10^{7} \mathrm{Nm}^{2}, 4.6 \times 10^{7} \mathrm{Nm}^{2}, 4.3 \times 10^{7}$ $\mathrm{Nm}^{2}, 4.0 \times 10^{7} \mathrm{Nm}^{2}$.

Figure $5 \mathrm{a}$ and $5 \mathrm{~b}$ show the trend of meshing stiffness in case of the changing the torsional stiffness from $K_{1}$ to $K_{5}$. As the torsional stiffness $K_{j}$ decreases, the meshing stiffness of the left side $s$ - $p$ meshing pairs increases, while the right meshing pair is opposite. When the torsional stiffness of the sun gear decreases from $5.2 \times 10^{7} \mathrm{Nm}^{2}$ to $4.0 \times 10^{7} \mathrm{Nm}^{2}$, the maximum meshing stiffness of left $s$ - $p$ meshing pairs is increases by $9.79 \%$, and the right side decreases by $11.23 \%$.

To analyze the influence of axial slide of the sun gear on time-varying meshing stiffness, left and right sides of $s-p$ meshing stiffness are calculated in case of $-0.5 \mathrm{~mm}, 0 \mathrm{~mm}$, $+0.5 \mathrm{~mm}$ and $+1 \mathrm{~mm}$, respectively. The direction of slide is indicated by ' + ' means sun gear slides towards the right (to output direction). The meshing stiffness of the $s-p$ meshing pairs under the sun gear axially sliding are shown in Figure 6.

According to Figure 6, when the sun gear slides $1 \mathrm{~mm}$ to the right under the initial torsional stiffness $K_{1}$, the maximum meshing stiffness of the left side $s$ - $p$ meshing 

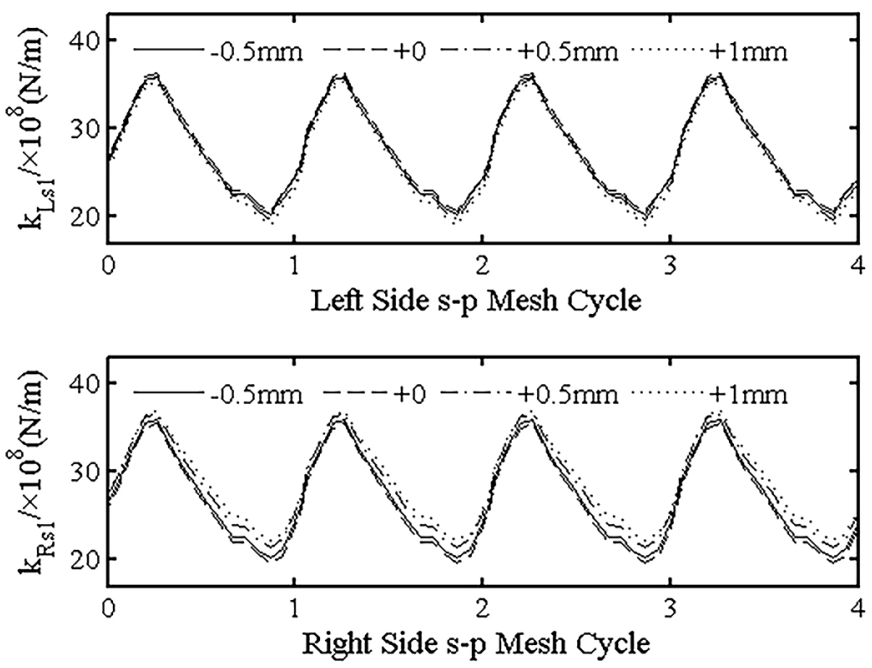

Fig. 6. Left and right sides meshing stiffness of $s-p$ mesh with sun gear axially sliding.

pairs decreases $6.30 \times 10^{7}(\mathrm{~N} / \mathrm{m})$, and the right side $s$ - $p$ meshing pairs increases $1.11 \times 10^{8}(\mathrm{~N} / \mathrm{m})$. From the point of view of time-varying meshing stiffness, when the sun gear slides axially, the time-varying meshing stiffness of $s-p$ meshing pairs could be adjusted by adjusting the torsional flexibility of the two sides teeth of the sun gear.

Then, according to equations (22) and (23), the dynamic gear meshing force time history for the left side and right side of $s-p 1$ meshing are obtained at mesh frequencies $1440 \mathrm{~Hz}$. When the torsional flexibility is rigid, the herringbone planetary gear can be considered to be completely symmetrical on the left and right, which is equivalent to spur gear. Parker's model [1] can be used to compare the dynamic meshing force to verify the model. For comparison, the dynamic load factor $L_{i s p}^{n}(t)$ is defined to represent the dynamic load sharing time history among the planets gear as equation (24).

$$
L_{i s p}^{n}(t)=4 F_{i s p}^{n}(t) / \sum_{n=1}^{4} F_{i s p}^{n}(t) .
$$

In fact, when torsional flexibility is not considered, the axial load of herringbone planetary gear offsets each other, and the model is very similar to the Parker's spur planetary gear model. But in this model, the herringbone gear is equivalent to the double helical gears on the left and right sides, and the direction of helix angle is opposite. And due to the torsional flexibility of herringbone planetary gear, the dynamic mesh force of left and right single helical gears is different. Figure 7 show the dynamic load factor time history of $s-p$ mesh, including both sides $s$ - $p 1$ mesh and $s-p 1$ mesh of equivalent Parker's model. From Figure 8 , the maximum dynamic load factor on the left is 1.04, and right side is 1.03 . They are slightly different, but are very close to 1.09 of Parker's model. In terms of the overall trend, Parker's model is very close to the dynamic load factor curve of $s-p$ on the right side of this article, and is slightly different from the left side. The results are a good agreement with the Parker's model results.

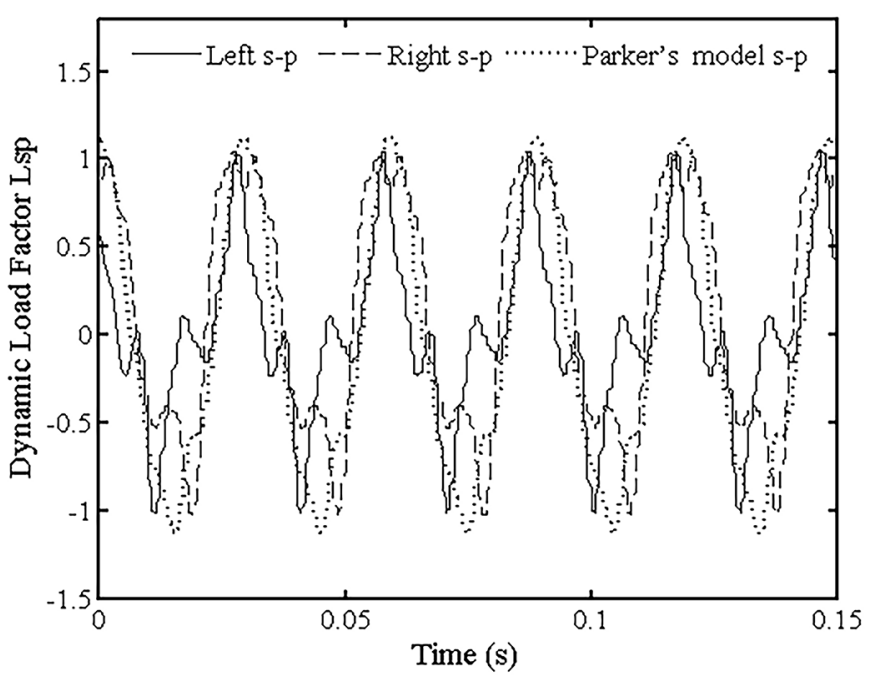

Fig. 7. Dynamic load factor time history of $s-p 1$ mesh.

\subsection{Influence of torsional flexibility on tooth surface load}

Tooth surface load is an important index that reflects the gear tooth meshing contact performance. References $[14,15]$ used the slice method in the axial direction for gears and analyzed the distribution of the tooth surface load of helical gears by considering various factors. The general expression for total load and total deformation of gear teeth can be obtained as:

$$
\int_{0}^{\tau}\left[K_{b}(x, \xi) p(\xi)+K_{c}(x, \xi) p(\xi)\right] d \xi=W
$$

where $K_{b}(x, \xi)$ and $K_{c}(x, \xi)$ represent the influence coefficient of the bending and contact deformation of the gear teeth, respectively. $p(\xi)$ represents the distributed load on the gear contact line, $W$ represents total meshing deformation, $\tau$ represents the total contact line length.

The sun gear tooth surface unit length load distributions are achieved for changing torsional stiffness from $K_{1}$ to $K_{5}$. Figure 8 shows the trend of unit length load of the tooth surface with torsional stiffness and axial sliding of sun gear.

According to Figure 8, the distributions of tooth surface load of the left and right $s-p$ meshing pairs are not completely the same. When the torsional stiffness $K_{1}$ equals $5.2 \times 10^{-5} \mathrm{rad}$, the maximum load area of the left $s-p$ meshing pairs is $880 \mathrm{~N} / \mathrm{mm}$, and the minimum load area is $260 \mathrm{~N} / \mathrm{mm}$, and the maximum load area of the right $s-p$ meshing pairs is $850 \mathrm{~N} / \mathrm{mm}$, and the minimum load is $270 \mathrm{~N} / \mathrm{mm}$. When the torsional stiffness $K 1$ equals $4.0 \times 10^{7} \mathrm{Nm}^{2}$, the maximum load area of the left $s-p$ meshing pairs is $940 \mathrm{~N} / \mathrm{mm}$, and the minimum load area is $280 \mathrm{~N} / \mathrm{mm}$, and the maximum load area of the right $s-p$ meshing pairs is $780 \mathrm{~N} / \mathrm{mm}$, and the minimum load area is $240 \mathrm{~N} / \mathrm{mm}$. The maximum load area of the left side $s-p$ meshing pairs increases by $6.82 \%$, but the right side decreases by $8.34 \%$. 


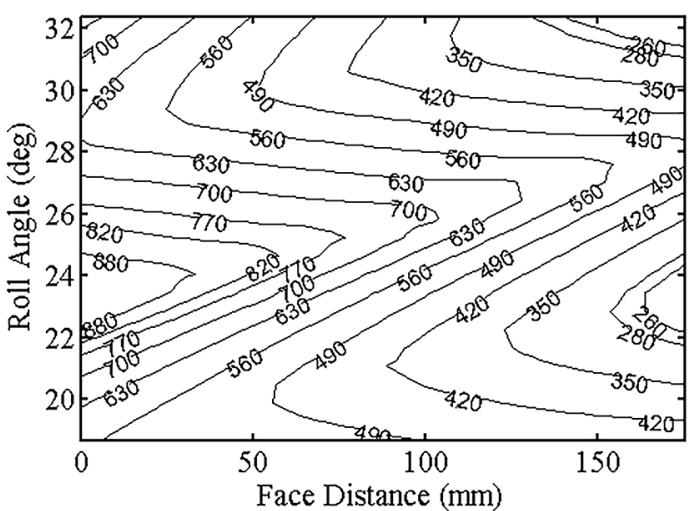

(a)

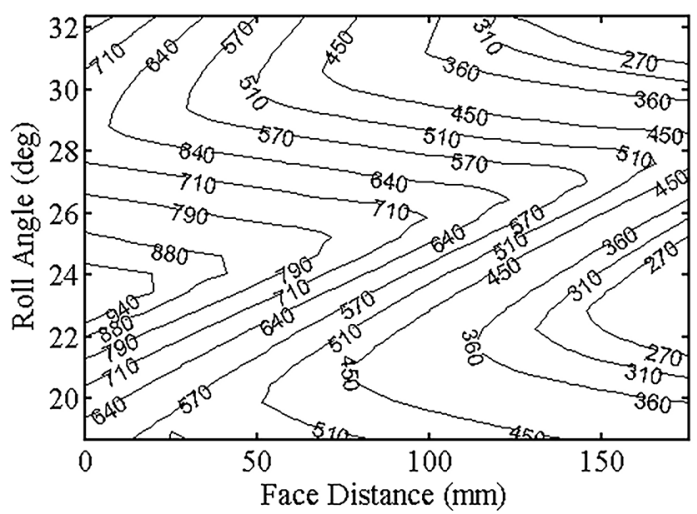

(c)

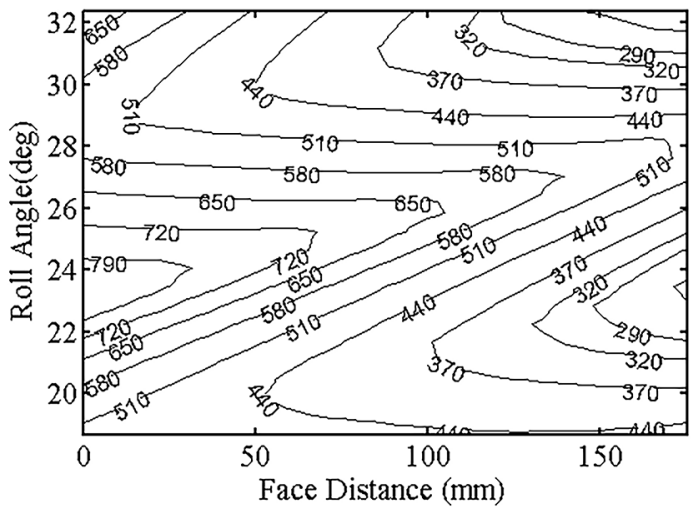

(e)

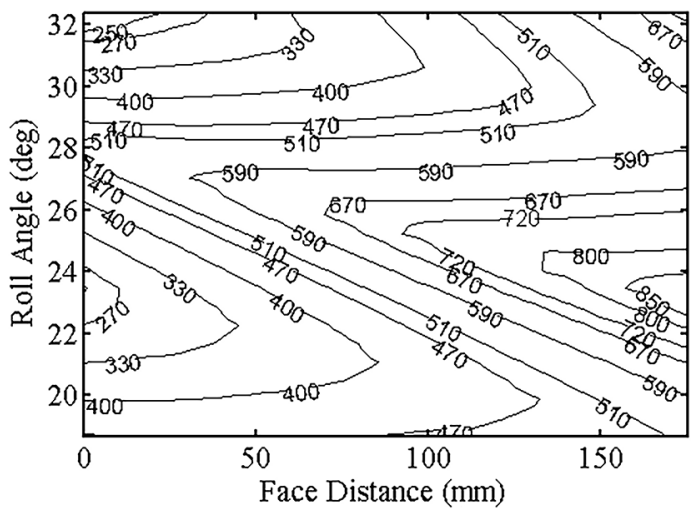

(b)

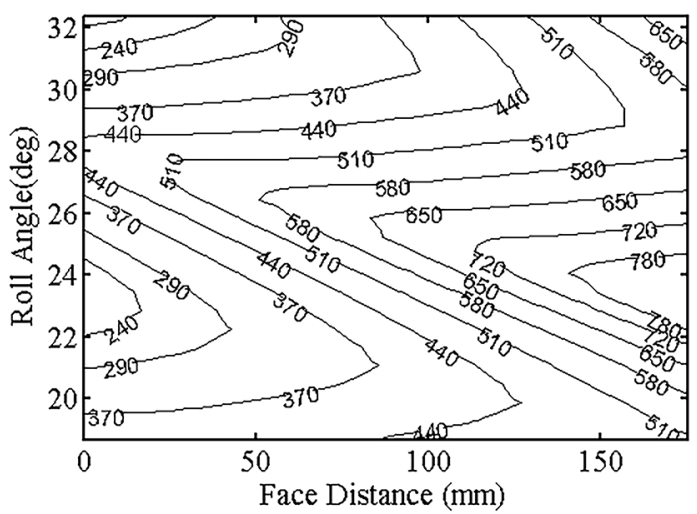

(d)

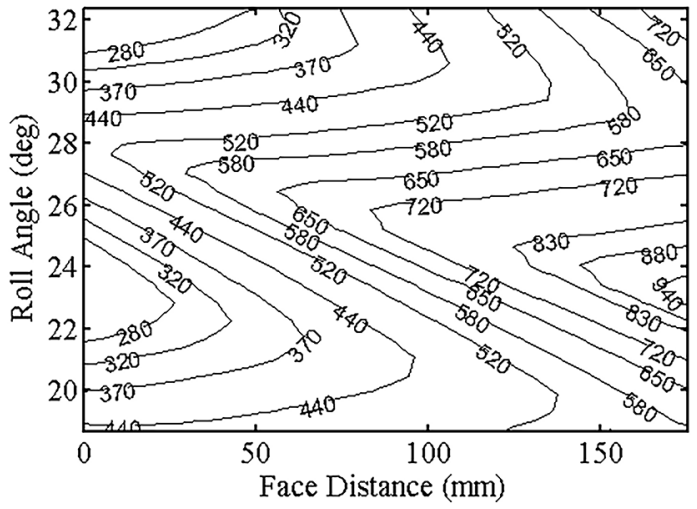

(f)

Fig. 8. Unit length load $(\mathrm{N} / \mathrm{mm})$ of tooth surface with torsional stiffness and axial sliding. (a) Left side with $K_{1}$. (b) Right side with $K_{1}$. (c) Left side with $K_{5}$. (d) Right side with $K_{5}$. (e) Left side with $+1 \mathrm{~mm}$ axially sliding and $K_{1}$. (f) Right side with $+1 \mathrm{~mm}$ axially sliding and $K_{1}$.

The same method can be used to obtain the tooth surface load distribution of the $s$ - $p$ meshing pairs under the sun gear sliding axially as shown in Figure 8e and 8f. When the sun gear slides to the right by $1 \mathrm{~mm}$ under the initial torsional stiffness $K_{1}$. The maximum load area of the left $s$ - $p$ meshing pairs is $790 \mathrm{~N} / \mathrm{mm}$, and the minimum load area is $250 \mathrm{~N} / \mathrm{mm}$, and the maximum load area of the right $s-p$ meshing pairs is $940 \mathrm{~N} / \mathrm{mm}$, and the minimum load area is $280 \mathrm{~N} / \mathrm{mm}$. The maximum load area of the left side $s-p$ meshing pairs decreases by $10.20 \%$, but the right side $s$ - $p$ meshing pairs increases by $10.59 \%$.

\section{Effects of torsional flexibility and axial sliding on dynamic mesh force}

In the HPGS, the dynamic mesh force is the main index of dynamic characteristics. Thus, the dynamic force response of the HPGS can be analyzed by changing torstional stiffness and axial sliding. Steady-state responses are obtained by using the fifth-order variable step-size RungeKutta method to solve the dynamic equation (22). Then the continuous frequency sweep method is used to analyze the dynamic mesh force response of the HPGS. 


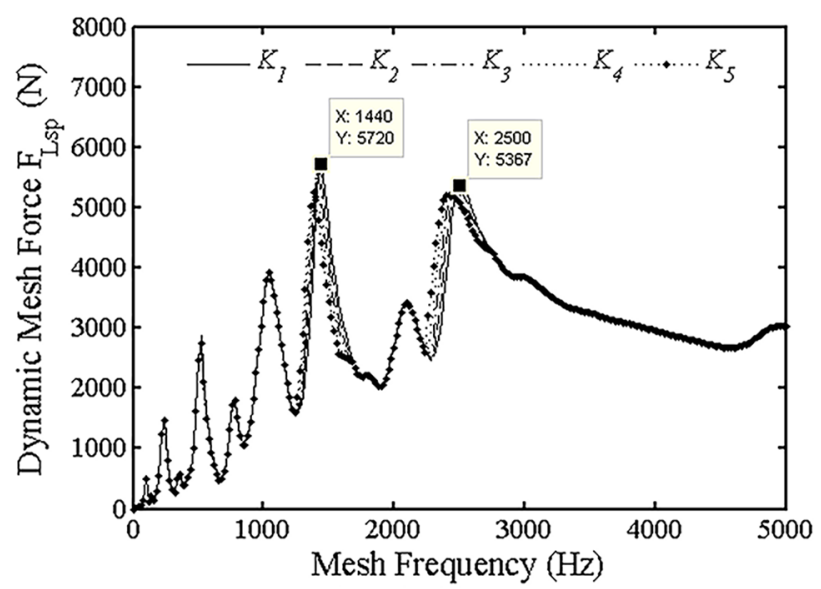

(a)

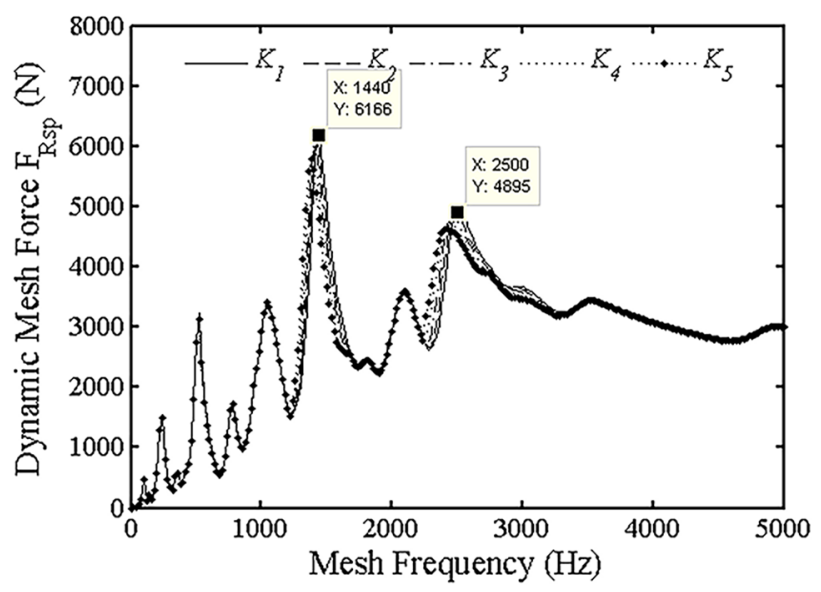

(b)

Fig. 9. Frequency sweep of dynamic mesh force with changing torsional stiffness. (a) Left side $s-p$ mesh. (b) Right side $s$ - $p$ mesh.

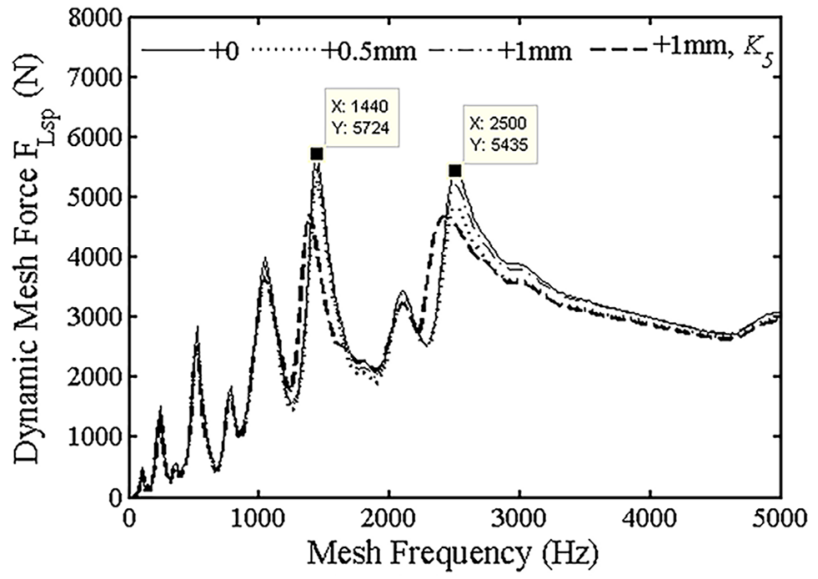

(a)

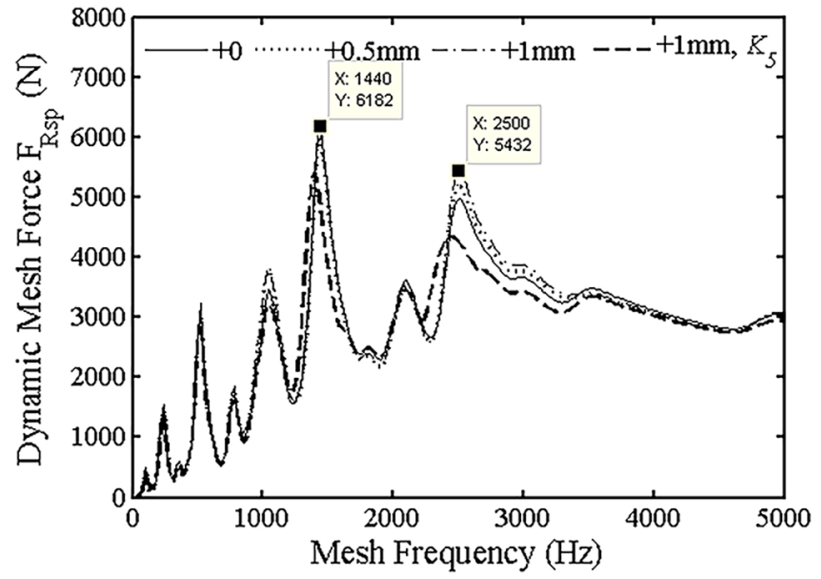

(b)

Fig. 10. Dynamic mesh force response under sun gear axially sliding. (a) Left side $s-p$ mesh. (b) Right side $s-p$ mesh.

In continuous frequency sweep, the frequency change is realized by continuous changing the meshing period. The steady-state response speed at the end of the previous sweep step is used as the initial condition for the next frequency sweep step. Here the amplitude of the response is the root-mean-square values of the dynamic mesh forces. At last, the left and right sides dynamic mesh force response $F_{i s p}$ of the $s-p$ mesh are conducted with different torsional stiffness and axial sliding of the sun gear.

Figure 9 shows the left and right sides dynamic mesh force response curves of the $s-p$ mesh are conducted by the $s-p$ mesh frequency sweep from $0 \mathrm{~Hz}$ to $5000 \mathrm{~Hz}$, and the torsional stiffness of sun gear is chosen from $K_{1}=5.2 \times 10^{7}$ $N \mathrm{~m}^{2}$ to $K_{5}=4.0 \times 10^{7} \mathrm{Nm}^{2}$. It can be seen from Figure 9a and $9 \mathrm{~b}$, the $s-p$ meshing pair has almost no change in dynamic mesh force response at low frequencies, and the resonance peak of the most obvious frequency change appears near the frequency of $1440 \mathrm{~Hz}$ and $2500 \mathrm{~Hz}$. With the increase of torsional flexibility, both the dynamic mesh force response peak values and peak frequencies of the left and right $s-p$ meshing pairs decrease very obviously. Therefore, appropriate increasing the torsional flexibility of the both sides tooth of the sun gear can reduce the main peak values and peak frequencies of the $s-p$ meshing pairs significantly, which will alleviate the vibration conditions of the system.

In order to observe the sun gear axially sliding on dynamic mesh force response, a group of axially sliding cases are employed to sweep the dynamic mesh force response. These axially sliding cases include $0 \mathrm{~mm}$, $+0.5 \mathrm{~mm},+1 \mathrm{~mm}$ under initial torsional stiffness $K_{1}$, and $+1 \mathrm{~mm}$ under torsional stiffness $K_{5}$, respectively. The dynamic mesh force response of the $s-p$ meshing pairs with the sun gear axially sliding can be shown in Figure 10.

According to Figure 10, when sun gear axially sliding changes from 0 to $+1 \mathrm{~mm}$ to the right side, the left dynamic mesh force response peak decreases and the right response peak increases. The most obvious frequency change appears near the frequency of $1440 \mathrm{~Hz}$ and $2500 \mathrm{~Hz}$. But both sides peak frequencies have almost no change when only axial sliding is applied. In addition, axial sliding and torsional stiffness are both applied, both sides response peak and response peak frequencies are still obviously decreased. 


\section{Conclusion}

This investigation takes the HPGS as the research object, mainly analyzing influence of the meshing stiffness, the tooth surface load and the dynamic mesh force of the HPGS under the changing of the torsional stiffness and the axial slide of the sun gear. Combining the structural characteristics of herringbone gears, a novel dynamic model of the HPGS is developed based on a lumped parameter method. The conclusions show that, as the torsional stiffness of the both sides tooth of sun gear decreases, the meshing stiffness and maximum load area of the left side $s-p$ meshing pair increase and the right side decrease, the dynamic mesh force response peak values of the left and right $s-p$ meshing pairs in all frequencies decrease, and peak frequencies of the main peak values decreases. When the sun gear slides to the right, the meshing stiffness, maximum load area and dynamic mesh force of the left side $s-p$ meshing pairs decrease, while the right side $s$ - $p$ meshing pairs increase, but peak frequencies keep the same. The results could be applied to reduce vibration and impact of the system, prolong the service life.

\section{Nomenclature}

$\mathrm{C}_{b} \quad$ The support damping matrix

$\mathrm{C}_{m} \quad$ The meshing damping matrix

$c_{i s 5} \quad$ The support damping of the sun gear on the $\zeta$ direction

$c_{i s n} \quad$ The meshing damping of the $n$th sun to planet meshing pairs

e Transmission error

E Elastic modulus

$F(t) \quad$ The dynamic exciting force

$F_{\text {irp }} \quad$ Dynamic mesh force of the $n$th ring to planet meshing pairs

$F_{i s p} \quad$ Dynamic mesh force of the $n$th sun to planet meshing pairs

G Gyroscopic matrix

$G_{j} \quad$ Modulus of elasticity in shear

I Moment of inertia

$I_{j} \quad$ Torsional moment of inertia

$I_{L s} \quad$ Moment of inertia of the left single helical gears of the sun gear

$I_{R s} \quad$ Moment of inertia of the right single helical gears of the sun gear

$\mathbf{K}_{b} \quad$ The matrix of support stiffness

$\mathbf{K}_{m} \quad$ The meshing stiffness matrix

$\mathbf{K}_{\omega} \quad$ The centripetal stiffness matrix

$K_{i e} \quad$ The stiffness matrix of beam element

$K_{h z} \quad$ The stiffness of Hertz contact

$K_{j} \quad$ Torsional stiffness between the two sides teeth of sun gear

$K_{m} \quad$ The meshing stiffness of meshing single pair

$K_{b}, K_{s}, K_{a}$ The stiffness of deformation of bending, shear and axial, respectively

$K_{b}(x, \xi) \quad$ The gears bending deformation coefficient

$K_{c}(x, \xi) \quad$ The gears contact deformation coefficient

$k_{0}$

$k_{\text {LRs }}$

$k_{i s n}$

$k_{\text {is }}$

$k(t)$

$L(t)$

l

$l_{L R}$

$M_{i e}$

$M$

m

$m_{L s}$

$m_{R s}$

$N$

$N_{r}, \quad N_{s}$

$n$

$n_{1}$

$O_{L}, O_{R}$

$p_{n}$

$p_{b}$

$p(\xi)$

$q$

$q_{h}$

$r-p$

$r_{1}$

$r_{c}$

$r_{b \zeta}$

$s-p$

$T$

$T_{D}$

$T_{m}$

$T_{s}$

$\Delta t$

$t$

$v$

W

$x$

$y$

$z$

$z_{s}$

$\alpha_{n}$

$\beta_{i b}$

$\delta$

$\varphi$

$\theta$

$\psi$
The stiffness in the $\zeta$ direction between the single helical gears on both sides of the sun gear

The meshing stiffness of the sun to $n$th planet The support stiffness of the sun gear on direction $\zeta$

Tooth meshing stiffness

The instantaneous total contact line length of single helical gear pair in herringbone gear

The length of the shaft segment from torsion center

Length of beam element

The mass matrix of torsion beam element Matrix of Mass

Mass

The mass of the left single helical gears of the sun gear

The mass of the right single helical gear of the sun gear

Number of meshing teeth pairs

Meshing pitch point of $r-p_{n}$ and $s-p_{n}$, respectively

Number of planet gear

The speed of the driving gear

Beam element nodes

The $n$th planet gear

The planetary gear pitch

The load distribution on contact line

Forced vibration response vector

Forced vibration response vector of the beam element

Meshing pair of ring gear to planet gear

The base circle radius of the driving gear

The distance from the center of planet gear to rotation center of carrier

The base circle radius of gear $\zeta\left(\zeta=s, r, p_{n}\right)$

Meshing pair of sun gear to planet gear

Torque

The input and output torque

The mesh period of all phases

The external load on the sun gear

The time interval of the $n$th to the first $s-p$ meshing pair

Time difference

Speed of meshing area

The whole meshing bending

The $x$ direction displacement of the component

The $y$ direction displacement of the component

The $z$ direction displacement of the component

Tooth number of sun gear

Position angle for planet $n$

Helix angle

Relative mesh displacement

Transverse pressure angle

The angular displacement of the component

Angle made by plane of action with vertical $y$ axis

Relative torsion angle 


$\begin{array}{ll}\phi_{n} & \begin{array}{l}\text { Relative angle of } n \text {th planet gear on the right } \\ \text { rotates to the position of the first planet gear }\end{array} \\ \omega_{c} & \text { Carrier angular rate } \\ \omega_{s} & \text { Sun gear angular rate } \\ \omega_{m} & \text { Mesh frequency } \\ \lambda & \text { Phase coefficient } \\ \tau & \text { The total contact line length }\end{array}$

\section{Subscripts}

\section{c Carrier}

$h$ The $h$ th node of herringbone gear middle segment torsion beam element

$i \quad$ Left or right side of herringbone gear $(i=\mathrm{L}, \mathrm{R})$

$j$ A value of torsional flexibility or torsional stiffness $(j=1,2,3,4,5)$

$p$ Planet gear

$r \quad$ Ring gear

$s$ Sun gear

$L \quad$ Left side of herringbone gear

$R$ Right side of herringbone gear

$x \quad x$ direction

$y \quad y$ direction

$z \quad z$ direction

5 Translational along $x, y$ and $z$ axis or angular displacement around the $z$ axis

This research is supported by the Foundation of the National Key Research and Development Plan of China (No. 2018YFB2001300), the Foundation of National Natural Science of China (No. 51975078, No. 51975079) and the Natural Science Foundation Project of Chongqing (No. cstc2018jcyjAX0087). Scientific Research Key Fund of Chongqing Municipal Education Commission (No. KJZD-K202000703).

\section{References}

[1] J. Lin, R.G. Parker, Analytical characterization of the unique properties of planetary gear free vibration, J. Vib. Acoust. 15, 316-321 (1999)

[2] L.L. Zhang, R.P. Zhu, Impact of meshing phase on load sharing for herringbone planetary train, J. Mech. Eng. 54, 129-140 (2018)

[3] P. Sondkar, A. Kahraman, A dynamic model of a doublehelical planetary gear set, Mech. Mach. Theory 157-174 (2013)

[4] Z.H. Bu, G. Liu, L.Y. Wu, Modal analyses of herringbone planetary gear train with journal bearings, Mech, Mach. Theory 54, 99-115 (2012)
[5] C.C. Zhu, X.Y. Xu, H.J. Liu et al., Research on dynamical characteristics of wind turbine gearboxes with flexible pins, Renew. Energy 68, 724-732 (2014)

[6] C.C. Zhu, X.Y. Xu, T.C. Lim et al., Effect of flexible pin on the dynamic behaviors of wind turbine planetary gear drives, Proc. Inst. Mech. Eng. C 227, 74-86 (2013)

[7] C. Wang, S.R. Wang, G.Q. Wang, Research on dynamic model of double helical gear pair based on TCA and LTCA, Int. J. Acoustics Vib. 24, 476-484 (2019)

[8] F. Wang, X. Xu, Z.D. Fang et al., Design and analysis of herring-bone gear with sixth-order transmission error based on meshing vibration optimization, Adv. Mech. Eng. 9 (2017)

[9] S. Mo, Y.D. Zhang, Q. Wu et al., Research on natural characteristics of double-helical star gearing system for GTF aero-engine, Mech. Mach. Theory 106, 166-189 (2016)

[10] S. Mo, T. Zhang, G.G. Jin et al., Dynamic characteristics and load sharing of herringbone wind power gearbox, Math. Probl. Eng. (2018)

[11] C.Z. Liu, D.T. Qin, T.C. Lim et al., Dynamic characteristics of the her-ringbone planetary gear set during the variable speed process, J. Sound Vib. 333, 6498-6515 (2014)

[12] F. Ren, G.F. Luo, G.Q. Shi, Influence of manufacturing errors on dynamic floating characteristics for herringbone planetary gears, Nonlinear Dyn. 93, 361-372 (2018)

[13] Z. Chen, Y. Shao, Meshing stiffness calculation of a spur gear pair with tooth profile modification and tooth root crack, Mech. Mach. Theory 62, 63-74 (2013)

[14] J.H. Wang, T.C. Lim, L.D. Yuan, Spur gear multi-tooth contact dy-namics under the influence of bearing elasticity and assembly errors, Proc. Inst. Mech. Eng. C 227, 2240-2455 (2013)

[15] Y. Miyoshi, K. Tobisawa, K. Saiki, Composite analysis method of tooth contact load distribution of helical gear, in ASME 2007 Inter-national Design Engineering Technical Conferences and Com-puter and Information in Engineering Conference, American So-ciety of Mechanical Engineers, 2013, 173-180

[16] M.B. Sanchez, J.I. Pedrero, M. Pleguezuelos, Contact stress calcu-lation of high transverse contact ratio spur and helical gear teeth, Mech. Mach. Theory 64, 93-110 (2013)

[17] C.G. Cooley, R.G. Parker, A review of planetary and epicyclic gear dynamics and vibrations research, Appl. Mech. Rev. 66, 040804 (2014)

[18] P. Sondkar, Dynamic modeling of double-helical planetary gear sets, The Ohio State University, Athens, 2012

[19] B. Yuan, S. Chang, G. Liu, L. Liu, L.D. Jiang, Analysis of dynamic characteristics of herringbone gear system considering cumulative error of tooth pitch, Vib. Shock 39, 120-112 (2020)

Cite this article as: X. Xu, H. Ge, J. Deng, J. Wang, R. Chen, An investigation on dynamic characteristics of herringbone planetary gear system with torsional flexibility between the left and right teeth of the sun gear, Mechanics \& Industry 21, 602 (2020) 